\title{
Multi-symplectic Spectral Methods for the Sine-Gordon Equation
}

\author{
A.L. Islas and C.M. Schober \\ Department of Mathematics and Statistics, Old Dominion University \\ Department of Mathematics, University of Central Florida \\ cschober@mail.ucf.edu
}

\begin{abstract}
Recently it has been shown that spectral discretizations provide another class of multi-symplectic integrators for Hamiltonian wave equations with periodic boundary conditions. In this note we develop multi-symplectic spectral discretizations for the sine-Gordon equation. We discuss the preservation of its phase space geometry, as measured by the associated nonlinear spectrum, by the multi-symplectic spectral methods.
\end{abstract}

\section{Introduction}

One approach to generalizing the concept of symplecticity to encompass partial differential equations (PDEs) is to develop a local concept of symplecticity that treats space and time equally [12/3/45]. A Hamiltonian PDE (in the " $1+1$ " case of one spatial and one temporal dimension) is said to be multi-symplectic (MS) if it can be written as

$$
\boldsymbol{M} z_{t}+\boldsymbol{K} z_{x}=\nabla_{z} S(z), \quad z \in \mathbb{R}^{d},
$$

where $\boldsymbol{M}, \boldsymbol{K} \in \mathbb{R}^{d \times d}$ are skew-symmetric matrices and $S: \mathbb{R}^{d} \rightarrow \mathbb{R}$ is a smooth function. The term MS is applied to system (11) in the sense that associated with $\boldsymbol{M}$ and $\boldsymbol{K}$ are the 2 -forms

$$
\omega(U, V)=V^{T} \boldsymbol{M} U, \quad \kappa(U, V)=V^{T} \boldsymbol{K} U, \quad U, V \in \mathbb{R}^{d},
$$

which define a symplectic space-time structure (symplectic with respect to more than one independent variable).

Symplecticity is a global property for Hamiltonian ODEs. In contrast, an important aspect of the MS structure is that symplecticity is now a local property, i.e., symplecticity may vary over the spatial domain and from time to time. This local feature is expressed through the following MS conservation law (MSCL):

$$
\partial_{t} \omega+\partial_{x} \kappa=0,
$$

where $U, V$ are any two solutions of the variational equation associated with (1)

$$
\boldsymbol{M} d z_{t}+\boldsymbol{K} d z_{x}=\boldsymbol{S}_{\boldsymbol{z z}}(z) d z .
$$


One consequence of multi-symplecticity is that when the Hamiltonian $S(z)$ is independent of $x$ and $t$, the PDE has an energy conservation law (ECL) [2]

$$
\frac{\partial E}{\partial t}+\frac{\partial F}{\partial x}=0, \quad E=S(z)-\frac{1}{2} z^{T} \boldsymbol{K} z_{x}, \quad F=\frac{1}{2} z^{T} \boldsymbol{K} z_{t},
$$

as well as a momentum conservation law

$$
\frac{\partial I}{\partial t}+\frac{\partial G}{\partial x}=0, \quad G=S(z)-\frac{1}{2} z^{T} \boldsymbol{M} z_{t}, \quad I=\frac{1}{2} z^{T} \boldsymbol{M} z_{x} .
$$

When the local conservation laws are integrated in $x$, using periodic boundary conditions, we obtain the global conservation of the total energy and total momentum.

MS integrators are discretizations of the PDE which preserve exactly a discrete version of the MSCL (2). In other words, MS integrators have been designed to preserve the MS structure, but not necessarily the local conservation laws or global invariants. Even so, numerical experiments using MS integrators, e.g., for the nonlinear Schrodinger (NLS) equation, have demonstrated that MS methods have remarkable conservation properties (cf. 4 45]). For example, we showed that the local and global energy and momentum are preserved far better than expected, given the order of the scheme. In addition, the global norm and momentum were preserved within roundoff.

However, the numerical experiments for the NLS equation demonstrated that MS finite difference schemes can have difficulty in resolving spatial structures in very sensitive regimes 4. On the other hand, spectral methods have proven to be highly effective methods for solving evolution equations with simple boundary conditions. As the number $N$ of space grid points increases, errors typically decay at an exponential rate rather than at polynomial rates obtained with finite difference approximations [7. For the NLS equation we showed that a significant improvement in the resolution of the qualitative features of the solution is obtained with a MS spectral method 6].

In this note we focus on the question of preservation of the phase space geometry of nonlinear wave equations by MS spectral methods. We use the sineGordon equation (SG) as our model equation. In the next section we present the MS formulation of the SG equation and a description of the phase space geometry in terms of the associated nonlinear spectrum. In section 3 we provide the MS spectral discretization of the SG equation. In section 4 we implement both MS and nonsymplectic spectral methods and use the nonlinear spectrum of the SG equation as a basis for comparing the effectiveness of the integrators. The relevant quantities to monitor are the periodic/antiperiodic eigenvalues of the associated spectral problem. These eigenvalues are the spectral representation of the action variables and are directly related to the geometry of the SG phase space. Significantly, we show that the MS spectral methods provide an improved resolution of phase space structures, as measured by the nonlinear spectrum, when compared with non-symplectic spectral integrators. 


\section{The MS and Integrable Structure of the SG Equation}

The MS form of the SG equation,

$$
u_{t t}-u_{x x}+\sin u=0
$$

is obtained by introducing the new variables $v=u_{t}, w=u_{x}$. This results in the system of equations

$$
\begin{aligned}
-v_{t}+w_{x} & =\sin u \\
u_{t} & =v \\
-u_{x} & =-w,
\end{aligned}
$$

which can be written in standard MS form (11) with

$$
z=\left(\begin{array}{c}
u \\
v \\
w
\end{array}\right), \quad \boldsymbol{M}=\left(\begin{array}{ccc}
0 & -1 & 0 \\
1 & 0 & 0 \\
0 & 0 & 0
\end{array}\right), \quad \boldsymbol{K}=\left(\begin{array}{ccc}
0 & 0 & 1 \\
0 & 0 & 0 \\
-1 & 0 & 0
\end{array}\right)
$$

and Hamiltonian $S(z)=-\cos u+\frac{1}{2}\left(v^{2}-w^{2}\right)$.

The energy and flux are given by

$$
\begin{aligned}
& E=S-\frac{1}{2} z^{T} \boldsymbol{K} z_{x}=-\cos u+\frac{1}{2}\left(v^{2}-w^{2}-u w_{x}+u_{x} w\right), \\
& F=\frac{1}{2} z^{T} \boldsymbol{K} z_{t}=\frac{1}{2}\left(u w_{t}-u_{t} w\right),
\end{aligned}
$$

respectively. Deriving relations (3)-(4) for the SG equation, the (ECL) can be simplified to

$$
E_{t}+F_{x}=\left(-\cos u+\frac{1}{2}\left(v^{2}+w^{2}\right)\right)_{t}-(v w)_{x}=0
$$

Similarly, the momentum conservation law is given by

$$
I_{t}+G_{x}=\left(\cos u+\frac{1}{2}\left(v^{2}+w^{2}\right)\right)_{x}-(v w)_{t}=0 .
$$

\subsection{Integrable Structure of the SG Equation}

The phase space of the SG equation (15) with periodic boundary conditions can be described in terms of the Floquet spectrum of the following linear operator (the spatial part of the associated Lax pair [9]):

$$
\mathcal{L}(u, \lambda)=\left[\boldsymbol{A} \frac{d}{d x}+\frac{i}{4} \boldsymbol{B}\left(u_{x}+u_{t}\right)+\frac{1}{16 \lambda} \boldsymbol{C}-\lambda \boldsymbol{I}\right]
$$

where

$$
\boldsymbol{A}=\left(\begin{array}{rr}
0 & -1 \\
1 & 0
\end{array}\right), \quad \boldsymbol{B}=\left(\begin{array}{ll}
0 & 1 \\
1 & 0
\end{array}\right) \quad \boldsymbol{C}=\left(\begin{array}{rr}
e^{i u} & 0 \\
0 & e^{-i u}
\end{array}\right), \quad \boldsymbol{I}=\left(\begin{array}{ll}
1 & 0 \\
0 & 1
\end{array}\right),
$$

$u$ is the potential and $\lambda \in \mathbb{C}$ denotes the spectral parameter. 
The fundamental solution matrix $M$, defined by the conditions $\mathcal{L}(u, \lambda) M=0$ and $M(x, x ; u, \lambda)=I$, is used to introduce the Floquet discriminant $\Delta(u ; \lambda)=$ $\operatorname{Tr}[M(x+L, x ; u, \lambda)]$. The Floquet discriminant is analytic in both its arguments. Moreover, for a fixed $\lambda, \Delta$ is invariant along solutions of the SG equation: $\frac{d}{d t} \Delta(u, \lambda)=0$. Since $\Delta$ is invariant and the functionals $\Delta(u, \lambda), \Delta\left(u, \lambda^{\prime}\right)$ are pairwise in involution, $\Delta$ provides an infinite number of commuting invariants for the SG equation.

The spectrum of $\mathcal{L}$ is given by the following condition on the discriminant: $\sigma(\mathcal{L})=\{\lambda \in \mathbb{C} \mid \Delta(u ; \lambda) \in \mathbb{R},-2 \leq \Delta(u ; \lambda) \leq 2\}$. When discussing the numerical experiments, we monitor the following elements of the spectrum which determine the phase space geometry of the SG equation: (a) Critical points $\lambda^{c}$, specified by the condition $d /\left.d \lambda \Delta(q ; \lambda)\right|_{\lambda=\lambda^{c}}=0$, and (b) Double points $\lambda^{d}$, which are critical points that satisfy the additional constraints $\left.\Delta(q ; \lambda)\right|_{\lambda=\lambda^{d}}= \pm 2$, $d^{2} /\left.d \lambda^{2} \Delta\right|_{\lambda=\lambda^{d}} \neq 0$. Complex double points correspond, in general, to critical saddle-like level sets and can be used to label their associated homoclinic orbits.

The periodic/antiperiodic spectrum provides the actions in an action-angle description of the system. The values of these actions fix a particular level set. Let $\lambda$ denote the spectrum associated with the potential $u$. The level set defined by $u$ is then given by, $\mathcal{M}_{u} \equiv\{v \in \mathcal{F} \mid \Delta(v, \lambda)=\Delta(u, \lambda), \lambda \in \mathbb{C}\}$. Typically, $\mathcal{M}_{u}$ is an infinite dimensional stable torus. However, the SG phase space also contains degenerate tori which may be unstable. If a torus is unstable, its invariant level set consists of the torus and an orbit homoclinic to the torus. These invariant level sets, consisting of an unstable component, are represented, in general, by complex double points in the spectrum. A complete and detailed description of the SG phase space structure is provided in [9].

\section{MS Spectral PDEs}

Bridges and Reich have shown that Fourier transforms leave the MS nature of a PDE unchanged [3]. A MS discretization of (1) is then obtained by truncating the Fourier expansion. This produces a system of Hamiltonian ODEs which can be discretized in time using symplectic methods. We briefly summarize these results.

Consider the space $L_{2}(I)$ of $L$-periodic, square integrable functions in $I=$ $[-L / 2, L / 2]$ and let $U=\mathcal{F} u$ denote the discrete Fourier transform of $u \in L_{2}(I)$. Here $\mathcal{F}: L_{2} \rightarrow l_{2}$ denotes the Fourier operator which gives the complex-valued Fourier coefficients $U_{k} \in \mathbb{C}, k=-\infty, \ldots,-1,0,1, \ldots, \infty$, which we collect in the infinite-dimensional vector $U=\left(\ldots, U_{-1}, U_{0}, U_{1}, \ldots\right) \in l_{2}$. Note that $U_{-k}=U_{k}^{*}$. We also introduce the $L_{2}$ inner product, which we denote by $(u, v)$ and the $l_{2}$ inner product, which we denote by $\langle U, V\rangle$. The inverse Fourier operator $\mathcal{F}^{-1}$ : $l^{2} \rightarrow L^{2}$ is defined by $\langle V, \mathcal{F} u\rangle=\left(\mathcal{F}^{-1} V, u\right)$. Furthermore, partial differentiation with respect to $x \in I$ simply reduces to $\partial_{x} u=\mathcal{F}^{-1} \Theta U$ where $\Theta: l_{2} \rightarrow l_{2}$ is the diagonal spectral operator with entries $\theta_{k}=i 2 \pi k / L$.

These definitions can be generalized to vector-valued functions $z \in L_{2}^{d}(I)$. Let $\hat{\mathcal{F}}: L_{2}^{d}(I) \rightarrow l_{2}^{d}$ be defined such that $Z=\left(Z^{1}, \ldots, Z^{d}\right)=\hat{\mathcal{F}} z=\left(\mathcal{F} z^{1}, \ldots, \mathcal{F} z^{d}\right)$. 
Thus with a slight abuse of notations and after dropping the hats, we have $Z=\mathcal{F} z, z=\mathcal{F}^{-1} Z$, and $\partial_{x} z=\left(\partial_{x} z^{1}, \ldots, \partial_{x} z^{d}\right)=\left(\mathcal{F}^{-1} \Theta Z^{1}, \ldots, \mathcal{F}^{-1} \Theta Z^{d}\right)=$ $\mathcal{F}^{-1} \Theta Z$.

Applying these operators to (1), one obtains an infinite dimensional system of ODEs

$$
\boldsymbol{M} \partial_{t} Z+\boldsymbol{K} \Theta Z=\nabla_{Z} \bar{S}(Z), \quad \bar{S}(Z)=\int_{-L}^{L} S\left(\mathcal{F}^{-1} Z\right) d x
$$

This equation can appropriately be called a MS spectral PDE with associated MSCL

$$
\partial_{t} \Omega+\Theta \mathcal{K}=0, \quad \Omega=\mathcal{F} \omega, \quad \mathcal{K}=\mathcal{F} \kappa
$$

and ECL

$$
\partial_{t} E+\Theta F=0, \quad E=\mathcal{F} e, \quad F=\mathcal{F} f
$$

in Fourier space.

\subsection{MS Spectral Schemes for the SG Equation}

A MS spatial discretization of the PDE is given by the truncated Fourier series,

$$
U_{k}=\frac{1}{\sqrt{N}} \sum_{l=1}^{N} u_{l} e^{-\theta_{k}(l-1) \Delta x}, u_{l}=u\left(x_{l}\right), x_{l}=-\frac{L}{2}+(l-1) \Delta x, \Delta x=\frac{L}{N},
$$

with

$$
\theta_{k}= \begin{cases}i \frac{2 \pi}{L}(k-1) & \text { for } k=1, \ldots, N / 2 \\ 0 & \text { for } k=N / 2+1 \\ -\theta_{N-k+2} & \text { for } k=N / 2+2, \ldots, N\end{cases}
$$

A family of discrete MSCLs exists which resemble the conservation law (7) in Fourier space 3 .

Using the discrete Fourier transform, system (6) becomes

$$
\begin{aligned}
-\partial_{t} V_{k}+\theta_{k} W_{k} & =\boldsymbol{F}_{k}(\sin u) \\
& =V_{k} \\
\partial_{t} U_{k} & =-W_{k} U_{k}
\end{aligned}
$$

This system of equations can be recombined into a single equation. In Fourier space we have that (8) becomes

$$
\ddot{U}_{k}=-\theta_{k}^{2} U_{k}-(\boldsymbol{F} \sin u)_{k}
$$

where

$$
(\boldsymbol{F} \sin u)_{k}=\frac{1}{N} \sum_{l=0}^{N-1} \sin u_{l} e^{-\theta_{k} l \Delta x}
$$

and the discrete Hamiltonian is given by 


$$
H=\frac{1}{2} \sum_{l=0}^{N-1}\left[\left|\dot{U}_{l}\right|^{2}+\mu_{l}^{2}\left|U_{l}\right|^{2}\right]-\frac{1}{N} \sum_{l=0}^{N-1} \cos u_{l} .
$$

To maintain the multi-symplecticity, a symplectic integrator in time should be used. To discretize (9) in time we note that the Hamiltonian (10) is separable which allows one to use explicit symplectic integrators. A general form of explicit higher order symplectic schemes is given by (see e.g., [10]),

$$
\begin{aligned}
a_{1} & =p_{j}, \quad b_{1}=q_{j}, \\
a_{i+1} & =a_{i}-C_{i} k V^{\prime}\left(b_{i}\right), \quad b_{i+1}=b_{i}+D_{i} k T^{\prime}\left(a_{i+1}\right), \quad i=1, \ldots, m, \\
p_{j+1} & =a_{m}, \quad q_{j+1}=b_{m},
\end{aligned}
$$

where the coefficients $C_{i}$ and $D_{i}$ are determined so that the scheme is symplectic and of order $O\left(k^{m}\right)$. For example, a first order scheme is given by $m=1$ and $C_{1}=1, \quad D_{1}=1$. Similarly, a second-order scheme is given by $m=2$ and $C_{1}=0, C_{2}=1, \quad D_{1}=\frac{1}{2}=D_{2}$. A fourth-order integrator $S_{4}$ can be obtained by forming the following product of second-order integrators $S_{2}$

$$
S_{4}(k)=S_{2}(\beta k) S_{2}(\alpha k) S_{2}(\beta k),
$$

where $\alpha=-2^{1 / 3} \beta$ and $\beta=1 /\left(2-2^{1 / 3}\right)$.

\section{Numerical Experiments}

The MS property can be lost in a discretization by using a non-symplectic discretization either in space or in time. Here we examine the loss of multisymplecticity due to the time discretization. We compare the performance of the spectral discretization implemented in time with second- and fourth-order symplectic methods (and thus MS) versus non-symplectic Runge-Kutta methods of the same order. In the numerical experiments we focus on determining whether the MS integrators preserve the structure of the SG phase space appreciably better than the nonsymplectic methods.

As derived using the inverse scattering theory [12, the SG equation has an infinite number of local conservation laws and global invariants. In this example, rather than monitor the local energy and momentum conservation laws, we choose to examine the preservation of the nonlinear spectrum, which incorporates all of the global invariants and determines the phase space structure.

Under the SG flow, the spectrum remains invariant. However, due to perturbations induced by the numerical discretization, the spectrum evolves in time. The evolution of the spectrum under the numerical flow is primarily due to the time discretization. To determine the effectiveness of MS spectral integrators in capturing the phase space structure, we compute the spectral content of the initial data and monitor its evolution under the different schemes.

The following initial data is used in the numerical experiments:

$$
u(x, 0)=\pi+0.1 \cos (\mu x), u_{t}(x, 0)=0,
$$


with parameters $\mu=2 \pi / L$ and $L=2 \sqrt{2} \pi$. This initial data is for solutions in the unstable regime as the zeroth double point remains closed, i.e. the initial data is on the level set containing the homoclinic manifold. (Closed double points cannot be preserved by the numerical schemes and in the following experiments one observes that the zeroth mode is immediately split into a gap state by the numerical scheme.)

To interpret the evolution of spectrum plots, note that under perturbations the complex double points can split in two ways - either into a gap along an arc of the circle, or into a cross along the radius (Figure 1). For each set of
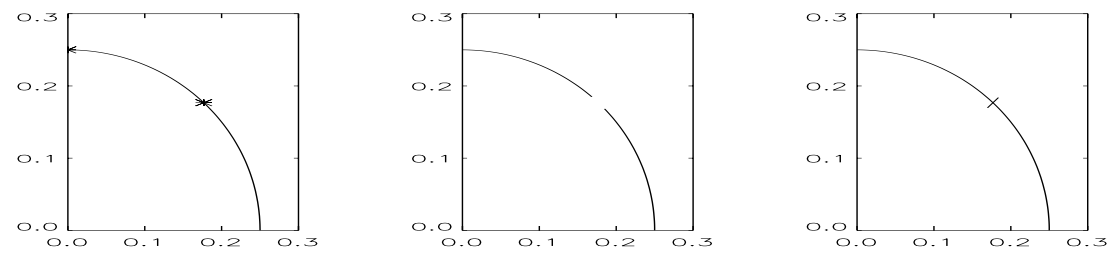

Fig. 1. The nonlinear spectrum. (a) Homoclinic orbit, (b) Inside the homoclinic orbit ('gap state'), (c) Outside the homoclinic orbit ('cross state').

experiments, we show a signed measure of the splitting distance for the zeroth mode as a function of time. Positive and negative values represent gap and cross states, respectively. When the splitting distance passes through zero, the double points coalesce and homoclinic crossings occur.

We consider the exponentially accurate spectral scheme (91) implemented in time with either Runge-Kutta (2nd and 4th-order) or with symplectic (1st, 2nd and 4th-order) integrators. These integrators will be denoted by S-2RK, S-4RK and S-1SY, S-2SY, S-4SY. In the spectral experiments we use $N=32$ Fourier modes and a fixed time step $\Delta t=L / 512$.

The splitting distance obtained with S-1SY is not shown but it is worth noting that even with the first-order symplectic integrator, bounded oscillations are observed. The splitting distance for both modes obtained with S-1SY is $O\left(10^{-2}\right)$. Using S-2RK and S-2SY (Figure 2), the spectrum for the first mode does not execute any homoclinic crossings for $0<t<500$ and so the torus component is accurately preserved. However, the zeroth mode does display homoclinic crossings which occur earlier than with the lower order S-1SY.

Since the initial data is chosen on the homoclinic manifold, it is to be expected that there will be an earlier onset and higher density of homoclinic crossings when a more accurate scheme is used. Refinement can accentuate the frequency of homoclinic crossings as the numerical trajectory is trapped in a narrower band about the homoclinic manifold. The main observation is that with the nonsymplectic S-2RK there is a $O\left(10^{-3}\right)$ linear drift in the error in the nonlin- 

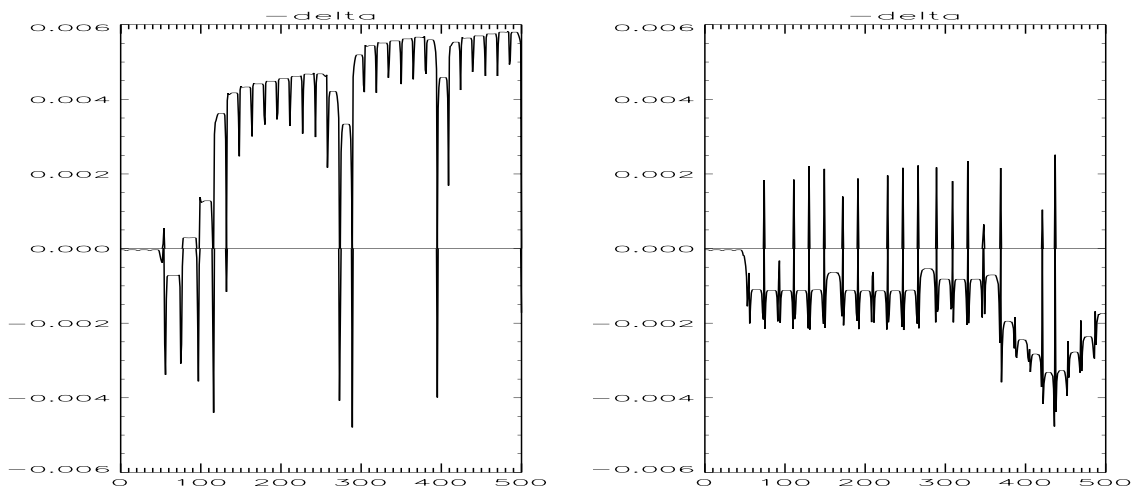

Fig. 2. Left: S-2RK: $u(x, 0)=\pi+0.1 \cos \mu x, u_{t}(x, 0)=0, N=32, t=0-500$. Right: S-2SY: $u(x, 0)=\pi+0.1 \cos \mu x, u_{t}(x, 0)=0, N=32, t=0-500$.

ear spectrum. The error in the nonlinear spectrum is smaller with S-2SY and further, it doesn't drift. The drift in the nonlinear spectrum obtained with S$2 \mathrm{RK}$ can be eliminated on the timescale $0<t<500$ by increasing the accuracy of the integrator and using S-4RK. In this case the nonlinear spectral deviations are $O\left(10^{-4}\right)$ for S-4RK and S-4SY (Figure 3). There does not seem to be an appreciable difference.
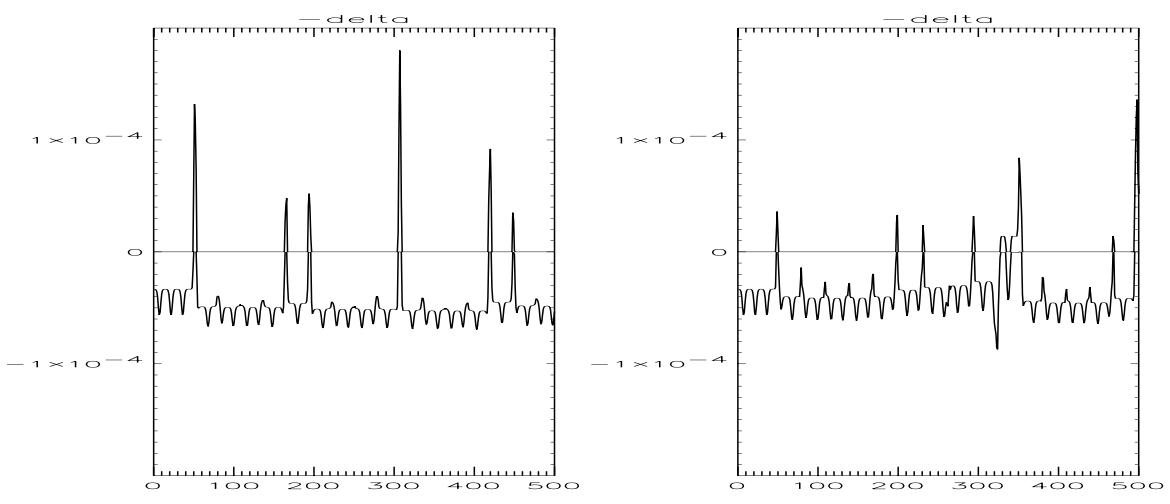

Fig. 3. Left: S-4RK: $u(x, 0)=\pi+0.1 \cos \mu x, u_{t}(x, 0)=0, N=32, t=0-500$. Right: S-4SY: $u(x, 0)=\pi+0.1 \cos \mu x, u_{t}(x, 0)=0, N=32, t=0-500$.

In long time studies of low dimensional Hamiltonian systems, symplectic integrators have been reported as superior in capturing global phase space structures since standard integrators may allow the actions to drift [811]. We continue the integration to $t=10,000$ and examine the time slice 10,000 $\leq t \leq 10,500$. For 
S-4RK (Figure 4) a drift has occured. The deviations in the actions associated with the zeroth mode oscillates about $1.2 \times 10^{-4}$ whereas for S-4SY (Figure 4) it oscillates about $5 \times 10^{-5}$. Although the drift observed with nonsymplectic schemes can be reduced by using a higher order integrator, it is not eliminated and simply occurs on a longer timescale. This problem is avoided using the MS integrator.

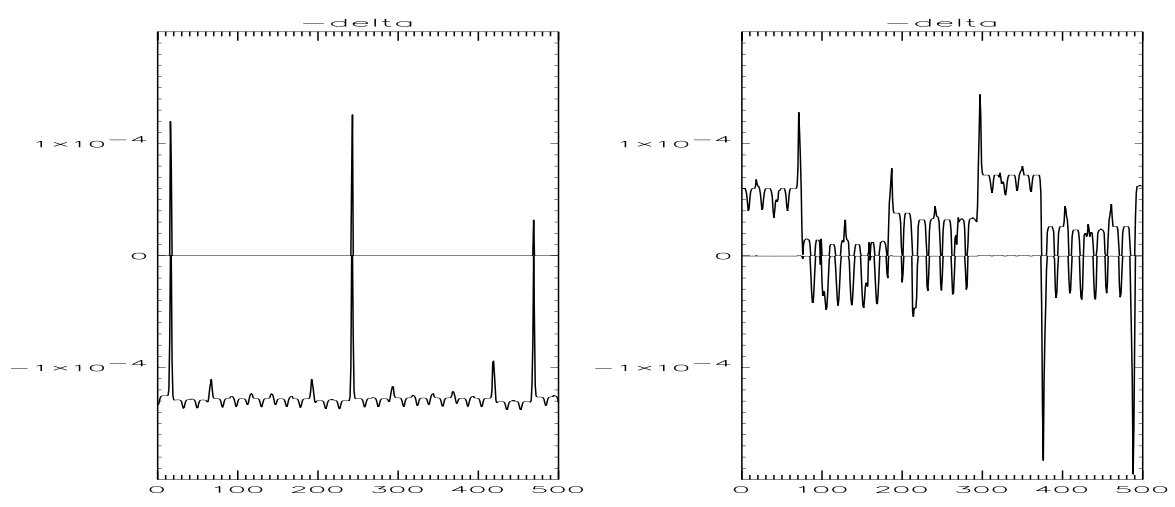

Fig. 4. Left: S-4RK: $u(x, 0)=\pi+0.1 \cos \mu x, u_{t}(x, 0)=0, N=32, t=10000-10500$. Right: S-4SY: $u(x, 0)=\pi+0.1 \cos \mu x, u_{t}(x, 0)=0, N=32, t=10000-10500$.

\section{Conclusions}

MS integrators are discretizations of the PDE which preserve exactly a discrete version of the MSCL. In this note, we have developed MS spectral integrators for the sine-Gordon equation. The benefits of these integrators are greater qualitative fidelity and superior preservation of local conservation laws and global invariants. The numerical experiments show that the MS spectral methods provide an improved resolution of phase space structures when compared with nonsymplectic spectral integrators.

\section{References}

1. Reich, S.: Multi-Symplectic Runge-Kutta Collocation Methods for Hamiltonian Wave Equations. J. of Comput. Phys. 157 (2000) 473-499

2. Bridges, T.J., Reich, S.: Multi-Symplectic Integrators: numerical schemes for Hamiltonian PDEs that conserve symplecticity. University of Surrey, Technical Report (1999)

3. Bridges, T.J., Reich, S.: Multi-Symplectic Spectral Discretizations for the Zakharov-Kuznetsov and shallow water equations. University of Surrey, Technical Report (2000) 
4. Islas, A.L., Karpeev, D.A., Schober,C.M.: Geometric integrators for the nonlinear Schrödinger equation. J. of Comp. Phys. 173 (2001) 116-148

5. Islas, A.L., Schober,C.M.: Multi-symplectic spectral methods for the GrossPitaevski equation. Lect. Notes Comp. Sci. 2331 (2002) 486-495

6. Islas, A.L., Schober,C.M.: Multi-symplectic methods for generalized Schrödinger equations. Fut. Gen. Comput. Sys. 950 (2003)

7. Fornberg, B.: A practical guide to pseudospectral methods. Cambridge University Press (1998)

8. Channell, P.J., Scovel, C.: Symplectic integration of Hamiltonian systems. Nonlinearity 3 (1990) 1-13

9. Ercolani, N., Forest, M.G., McLaughlin, D.W.: Geometry of the modulational instability, Part III. Physica D 43 (1990) 349-360

10. Yoshida, H.: Construction of higher order symplectic integrators. Phys Lett. A 150 (1990) 262-268

11. Sanz-Serna, J., Calvo, M.: Numerical Hamiltonian problems. Chapman and Hall, London (1994)

12. Ablowitz, M.J., Segur, H.: Solitons and the inverse scattering transform. SIAM Studies in Applied Math., SIAM, Philadelphia (1981) 